\title{
EFFECTS OF PNEUMOCOCCAL VACCINE IN PATIENTS WITH CHRONIC RESPIRATORY DISEASE
}

\author{
Yuji Watanuki, N. Miyazawa, M. Kudo, S. Inoue, H. Goto, H. Takahashi, T. Kaneko and Y. Ishigatsubo
}

Research Excellence Award in Respiratory Infections, sponsored by Cellestis

Respiratory Medicine, Yokohama City University School of Medicine, Yokohama, Japan

WINNING ABSTRACT: In developed countries, it is very difficult to demonstrate the effectiveness of pneumococcal vaccines because the incidence of pneumococcal pneumonia is very low.

Vaccination against pneumococci infection was advised for 1378 outpatients, over 60 years of age, with chronic respiratory disease for more than one year. Of these patients, those who responded affirmatively to the advice were vaccinated against pneumococci between August and November 2002. The effectiveness of vaccination was evaluated by means of a 2-year cohort-study, comparing the vaccinated group (647) with the non-vaccinated group (731). The variables analyzed were the frequency of onset of bacterial respiratory infection, hospitalization due to bacterial respiratory infection and onset of pneumococcal respiratory infection.

The incidence of bacterial respiratory infection and the incidence of pneumococcal respiratory infection to have decreased in the following 2 years $(17.4 \%, 0.9 \%)$, as compared to the previous year $(25.9 \%, 3.1 \%)$, in the vaccinated group. Conversely, the frequency was higher in the following 2 years $(14.4 \%, 0.9 \%)$ as compared to the previous year $(14.2 \%, 0.4 \%)$ in the nonvaccinated group. This inter-group difference was statistically significant. Simultaneous vaccination against pneumococci and influenza virus also resulted in a significant reduction in the incidence of bacterial respiratory infection. No decrease was observed in the frequency of hospitalization.

These results indicate that pneumococcal vaccine is useful for elderly patients with chronic respiratory disease and that its efficacy may be enhanced by simultaneous vaccination against influenza.

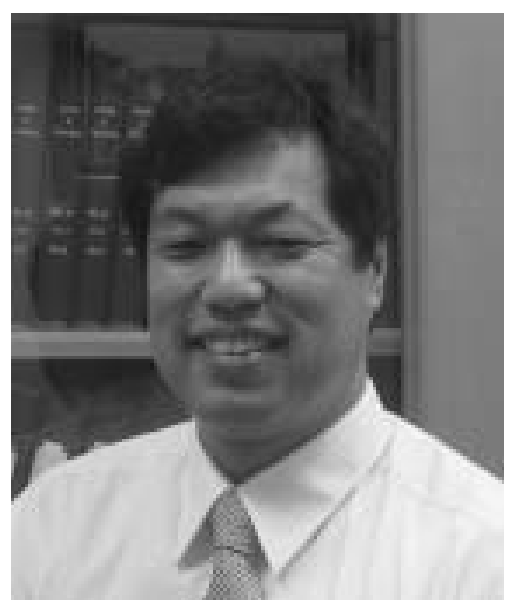

Yuji Watanuki

Dept of Respiratory Medicine, Yokohama City

University School of Medicine, Yokohama, Japan

\section{MY JOB AND THE UNIT IN WHICH I WORK}

I have worked for 11 yrs as a medical doctor in the pulmonary division of the Kanagawa Cardiovascular and Respiratory diseases Center, Yokohama, Japan. Since 2006, I have been Head of the Dept of Respiratory Medicine in Yokohama City University School of Medicine, Yokohama, involved in patient care, research, student education and special training. The present study was performed by the Clinical Study Team of the

STATEMENT OF INTEREST: None declared.
Kanagawa Cardiovascular and Respiratory Diseases Center, which is associated with the Yokohama City University. The institution is a large, specialised regional centre for pulmonology (170 in-patient beds), which each month treats 3,700 patients (200 in-patients and 3,500 outpatients) with respiratory diseases. The centre offers general respiratory care and a wide range of subspeciality services, including chronic respiratory infections, interstitial lung disease, lung cancer, tuberculosis and chronic respiratory failure. Respiratory research at the university is focused around difficult respiratory infections and is closely linked to clinical work in the associated hospitals where many patients with chronic respiratory diseases regularly receive outpatient care.

\section{MY WINNING ABSTRACT AS PART OF MY RESEARCH}

When I started my PhD with my thesis entitled "Correlation of Quantitative CT with selective alveolobronchogram and pulmonary function tests in emphysema" [1], the research performed in our dept was mainly focused on the pathogenesis and treatment of chronic obstructive pulmonary disease (COPD). Following my $\mathrm{PhD}$, my further research focused on control of breathing in patients with COPD [2, 3]. Part of my scientific work is the long-term observation of patients with chronic respiratory tract infection. In particular, I have investigated the antibiotic resistance according to genotype of penicillin-binding protein and macrolide resistance genes of Streptococcus peumoniae and Haemophilus influenzae isolated from the patients with chronic respiratory diseases. I identified the relationship between the emergence of resistant genes and the risk factors of the patients. The pneumococcal vaccine includes 23 purified capsular antigens, which covers $95 \%$ of the penicillin-nonsusceptible serotypes, and is expected to 


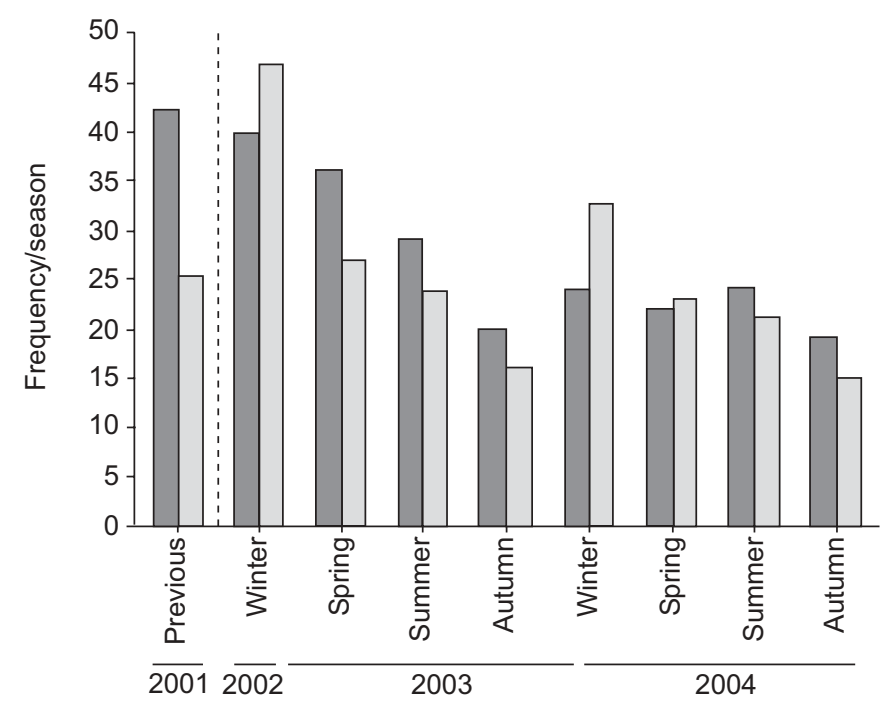

FIGURE 1. Changes in the frequency of bacterial respiratory infection (2-yr

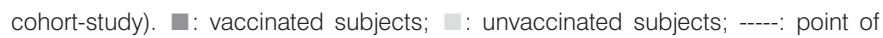
vaccination against pneumococci.

reduce the incidence of drug-resistant strains [4]. I then focused on the usefulness of the pneumococcal vaccine in preventing either pneumonia or death in adults with chronic respiratory disease and reducing penicillin-nonsusceptible strains.

The percentage of respiratory tract infections caused by pneumococcus is particularly high among elderly patients. The currently available pneumococcal vaccine stimulates the formation of specific antibodies in vivo, resulting in the prevention of pneumococcal infection; the antibody specific to pneumococci is produced within 1 month of vaccination and is retained in vivo for $\sim 5 \mathrm{yrs}$. Among the studies conducted to date to evaluate the effectiveness of pneumococcal vaccines, a placebo-controlled study involving mine workers (known to have a high incidence of pneumococcal pneumonia, i.e. several percent or higher per year) and some other groups was performed in the 1970s. The study demonstrated the effectiveness of vaccines against pneumonia and sepsis. In developed countries, however, it is very difficult to demonstrate the effectiveness of pneumococcal vaccines with this type of study because the incidence of pneumococcal pneumonia is very low and $>20,000$ subjects need to be assigned to both the vaccinated group and the control (nonvaccinated group) for evaluation of effectiveness. In recent years, the effectiveness of pneumococcal vaccines was studied primarily using case-control or indirect cohort study designs, demonstrating that vaccines are not useful in preventing the onset of nonbacteraemic pneumonia, but are for pneumococcal bacteraemia $[5,6]$. In Japan, the percentage of people vaccinated against pneumococcal infection is very low $(2 \%)$, and it is difficult to perform a case-control study involving subjects with pneumococcal infection, as is often done in developed countries. In our cohort study, we were able to demonstrate the usefulness of vaccination despite the relatively small size of the population studied. This probably owes much to the study design, i.e. the adoption of bacterial respiratory infection (which often complicates chronic respiratory disease) as an indicator of the effectiveness of vaccination and the technique of repeated measures analysis of variance, to compare the change in the incidence of infection from the pre-vaccination to the post-vaccination period between the vaccinated and nonvaccinated groups (fig. 1, table 1).

\section{MY RESEARCH AS PART OF MY WORKING GROUP/ RESEARCH TEAM}

The presentation in my winning poster fits well into the main research area of the First Dept (Immunology, Hematology and Respirology) of Medicine at the Yokohama City University School of Medicine, led by Professor Y. Ishigatsubo. Our team has been involved in many research projects. The main topics of a currently running research project include the following: the role of haem oxygenase 1 (Ho-1) in variable conditions; the resistant model of Pseudomonas aeruginosa; and difficult pulmonary infections in immunocompromised host.

\section{THE IMPACT OF MY WORK ON CLINICAL OR RESEARCH PRACTICE}

Although the primary purpose of pneumococcal vaccination is to prevent pneumococcal pneumonia, prevention of pneumonia in general (which is the fourth leading cause of death in Japan, second only to malignant neoplasm, cardiac disease and cerebrovascular disease, and is responsible for $8.6 \%$ of all deaths) is also among the ultimate goals. In the present study, we analysed not only pneumococcal pneumonia but also bacterial

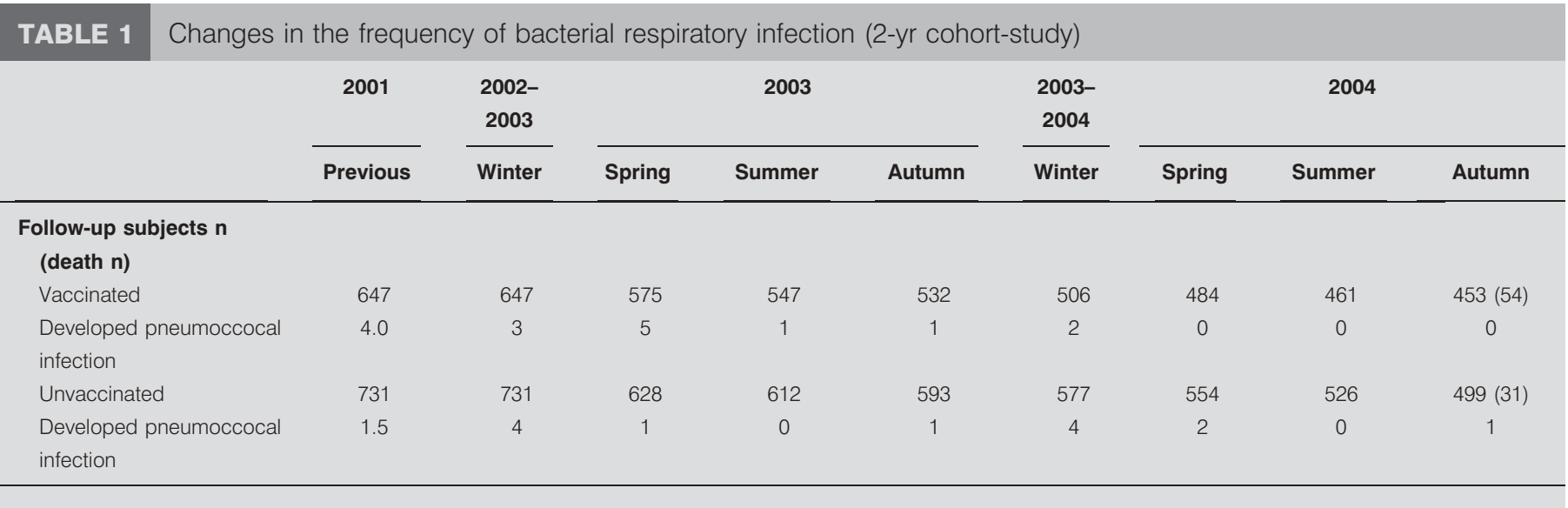


respiratory infection as an indicator of the effectiveness of vaccination and concluded that pneumococcal vaccine is useful for elderly patients with chronic respiratory disease. Our results indicate pneumococcal vaccination appears to protect against nonbacteraemic pneumonia and death in adults. It is possible that further recommendations will extend the use of pneumococcal vaccine to younger adults and patients at high risk. Consideration would be given to public funding of the pneumococcal vaccine for these people in countries with low vaccination rates, such as Japan. Further research is required to reveal the usefulness of the vaccine in preventing the increase of drug-resistant Streptococcus peumoniae.

\section{REFERENCES}

1 Watanuki Y, Suzuki S, Nishikawa M, Miyashita A, Okuba T. Correlation of quantitative CT with selective alveolobronchogram and pulmonary function tests in emphysema. Chest 1994; 106: 806-813.
2 Yoshiike Y, Suzuki S, Watanuki Y, Okubo T. Effect of fenoterol on ventilatory responses to hypoxia and hypercapnia in normal subjects. Thorax 1995; 50: 139-142.

3 Suzuki S, Watanuki Y, Yoshiike Y, Okubo T. Effect of fenoterol on ventilatory responses to hypercapnia and hypoxia in patients with chronic obstructive pulmonary disease. Thorax 1997; 52: 125-129.

4 Kyaw MH, Lynfield R, Schaffner W, et al. Effect of introduction of the pneumococcal conjugate vaccine on the drug-resistant Streptococcal pneumoniae. N Eng J Med 2006; 354: 1455-1463.

5 Bultier JC, Breiman RF, Campbell JF, Lipman HB, Broome CV, Facklam RR. Pneumococcal polysaccharide vaccine efficacy, an evaluation of current recommendations. JAMA 1993; 270: 1826-1831.

6 Jackson L, Neuzil K, Yu O, et al. Effectiveness of pneumococcal polysaccharide vaccine in older adults. $N$ Eng J Med 2003; 348: 1747-1755. 